\title{
Sexual Differences in Chimpanzee Sociality
}

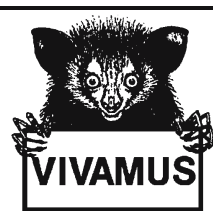

\author{
Julia Lehmann • Christophe Boesch
}

Received: 2 September 2005 /Revised: 24 April 2006 / Accepted: 17 November 2006 / Published online: 17 January 2008

(C) Springer Science + Business Media, LLC 2007

\begin{abstract}
Scientists usually attribute sexual differences in sociality to sex-specific dispersal patterns and the availability of kin within the social group. In most primates, the dispersing sex, which has fewer kin around, is the less social sex. Chimpanzees fit well into the pattern, with highly social philopatric males and generally solitary dispersing females. However, researchers in West Africa have long suggested that female chimpanzees can be highly social. We investigated whether chimpanzees in the Taï Forest (Côte d'Ivoire) exhibit the expected sexual differences in 3 social parameters: dyadic association, party composition, and grooming interactions. Though we found a significant sexual difference in each of the 3 parameters, with males being more social than females, the actual values do not reveal striking differences between the sexes and do not support the notion of female chimpanzees as asocial: females had dyadic association indices comparable to mixed-sex dyads, spent $c a$. $82 \%$ of their time together with other adult chimpanzees, and had a comparable number of grooming partners. Further, female associations can be among the strongest bonds within the community, indicating that both sexes can have strongly favored association partners. The findings are in contrast to reports on East African chimpanzees, the females of which are mainly solitary and rarely interact with other females. Our results suggest that researchers cannot generally regard chimpanzee females as asocial and need to redefine models deriving patterns of sociality from dispersal patterns to integrate the possibility of high female sociality in male philopatric systems.
\end{abstract}

J. Lehmann $\cdot$ C. Boesch

Max Planck Institute for Evolutionary Anthropology, 04103 Leipzig, Germany

J. Lehmann

School of Biological Sciences, University of Liverpool, Liverpool L69 7ZB, UK

Present address:

J. Lehmann $(\bowtie)$

School of Human \& Life Sciences, Roehampton University, London SW15 4JD, UK

e-mail: j.lehmann@roehampton.ac.uk 
Keywords association $\cdot$ chimpanzee $\cdot$ sex differences $\cdot$ sociality

\section{Introduction}

One consequence of group living is that individuals have to interact frequently with other group members. Such interactions can be either aggressive or affiliative, and researchers have shown great interest in both types (Huntingford and Turner 1987). The extent to which males and females engage in (same-sex) social interactions often differs dramatically, and scientists generally consider sexual differences in sociality as the result of competitive regimes to be the driving factor in the evolution of the social systems and dispersal patterns (Isbell and Young 2002; Sterck et al. 1997; van Schaik 1989; Wrangham 1980). Traditionally, researchers have attributed the link between dispersal patterns and the strength of intrasexual relationships (Harcourt and Stewart 1983; Smuts 1990; Wrangham 1980) to advantages arising from kin selection (Hamilton 1964), i.e., the benefits to both social partners can be enhanced if they are related to each other (Greenwood 1980; Waser 1988). Because philopatric individuals are more likely to be closely related, strong social bonds occur primarily between members of the philopatric sex. Further, the extended presence of the social partner over time is more reliable in the philopatric sex so that the philopatric sex is probably more likely to gain long-term benefits than the dispersing sex (Clutton-Brock 2002).

Overall, intergroup dispersal patterns seem to predict well intrasexual relationships among nonhuman primates: strong social bonds between females occur in many male-dispersing primates: macaques (Macaca mulatta: Kapsalis and Berman 1996), vervets (Cercophitecus aethiops: Seyfarth 1980), capuchins (Cebus capucinus: Perry 1996), savannah baboons (Papio cynocephalus ursinus: Seyfarth 1976; Silk et al. 1999), and gelada baboons (Theropithecus gelada: Dunbar 1984). In the few species in which females disperse and males remain in their natal communities stronger bonds often occur between males: red colobus (Colobus badius: Struhsaker and Leland 1976, cf. Starin 1994), chimpanzees (Pan troglodytes: Goodall 1986; Watts 2000a,b) and hamadryas baboons (Papio hamadryas: Kummer 1968, cf. Swedell 2002). Thus, most primate societies seem to conform to the general predictions of the models of evolution of primate social organization (Sterck et al. 1997). However, the models also predict that there are only weak or no bonds between the members of the dispersing sex, which seems to be an oversimplification. Some exceptions are the female howlers (Pope 2000) and bonobos (Hohmann et al. 1999; Kano 1992; Parish and De Waal 2000), which form strong bonds, though they disperse from their natal groups.

Chimpanzees (Pan troglodytes) have been reported to generally fit the model, in that they show strong social bonds between the philopatric males and only weak social relations among the dispersing females (Arnold and Whiten 2003; Goodall 1986; Nishida 1979; Wrangham et al. 1992). However, as more data on female chimpanzee sociality accumulate, it has become evident that their social behavior varies considerably between study sites (Boesch and Boesch-Achermann 2000; Fawcett 2000; Wakefield 2002; Williams et al. 2002b). Findings from several East African chimpanzee study sites such as Gombe (Goodall 1986: Williams et al. 
2002b), Kibale (Wrangham et al. 1992), and Mahale (Kawanaka 1984) are generally in line with the view of low female sociality, but reports from other populations suggest that females may be far more social than previously reported (Boesch and Boesch-Achermann 2000; Fawcett 2000; Ghiglieri 1984; Wakefield 2002), thereby contradicting the generally assumed link between dispersal patterns and sociality. Sex differences in social behavior should arise if the advantages gained through that behavior are much higher in one sex than in the other. In chimpanzees, where males defend the community home range, have clear linear dominance hierarchies, and obtain the highest reproductive success when dominant (Boesch and BoeschAchermann 2000; Nishida et al. 1985; Watts and Mitani 2001; Williams et al. 2002a), they are expected to also profit strongly from cooperation and alliances. Conversely, females compete primarily over food and may avoid each other in order to reduce competition. However, recent studies suggest that chimpanzee females can experience strong competition, which could favor the formation of social bonds among them. For example, findings from Gombe indicate that female rank and female competition for space, and presumably food, are important determinants of female reproductive success (Williams et al. 2002a, 2004), though strong social bonds between unrelated females did not occur (Williams et al. 2002b). Taï chimpanzee females possess a linear dominance hierarchy (Wittig and Boesch 2003), cooperate to obtain access to monopolizable food sources (Boesch and BoeschAchermann 2000; Wittig, pers. comm.), and effectively support males in their fights for dominance and take part in home range defense activities (Boesch and BoeschAchermann 2000). Thus, having cooperative partners or allies should be important for both male and female chimpanzees. Further, the assumption that kin selection is a major driving force for social behavior in chimpanzees is weakened by recent findings: average relatedness between chimpanzee males is not significantly higher than it is between females (Vigilant et al. 2001, cf. Morin et al. 1994) and males do not seem to cooperate preferentially with related partners (Mitani et al. 2000). Thus, the observed patterns of intrasexual affiliation/cooperation in chimpanzees appear to be driven by factors other than kinship (Parish and De Waal 2000; Williams et al. $2002 \mathrm{~b}$ ), and it seems evident that, contrary to the notion of their asocial behavior, chimpanzee females could benefit from sociality.

We analyzed the extent to which a group of West African chimpanzees studied for $>10 \mathrm{yr}$ exhibit sexual differences in social behavior. Specifically, we tested if the often reported generalizations about chimpanzee sociality hold for Taï chimpanzees: 1) males spend most of their time in parties, while females are mostly alone, 2) males have a higher dyadic association index than females, and 3) males invest more into grooming relationships than females do.

\section{Methods}

\section{Study Site and Data Collection}

The Taï National Park, Côte d'Ivoire, West Africa, comprises an area of $c a .4540 \mathrm{~km}^{2}$ and consists of evergreen lowland rain forest (Boesch and Boesch-Achermann 2000). Our data are from the North community, habituated in 1979, which researchers and 
field assistants have since observed continuously. From 1992 onwards, we carried out daily focal individual follows (Altmann 1974), and field assistants, Honora Néné Kpazahi and Grégoire Kohou Nohon, continuously collected data on party size and composition, ranging behavior, and social interactions of the target individual on checksheets. We usually followed targets from the first activity in the morning to nesting at night or as long as possible (mean \pm standard deviation of daily observation time: $9.7 \pm 2.1 \mathrm{~h}$ ). We usually located targets by either following their vocalizations or by choosing a different member of a nesting party from a known location, aiming at following all adult individuals at least once per month. Data provided here encompass $\leq 10 \mathrm{yr}$, from January 1992 to December 2001, during which community size decreased steadily, leading to an increasingly female-biased sex ratio (Table I). We observed each chimpanzee included into subsequent analyses for 13.4 7.5 (mean \pm $\mathrm{SD}) \mathrm{d} / \mathrm{yr}$. The large SD value is due to the decrease in community size, i.e., when the community became smaller we could follow each individual more often. We collected a total of 515 follows on males and 1447 follows on females. We chose targets in a semirandomized way, with the condition of following each adult individual at least once per month. Because there is no datum on male follows for 1993 and 1994, data on male-male interactions are based on 8 yr of observation only, excluding 1993/94. However, we could still calculate dyadic association indices for the full 10 -yr period for both sexes because they are based purely on the presence or absence of individuals in parties. Because Taï chimpanzees often move in large, mixed-sex parties, we observed males sufficiently frequently to calculate association indices also for 1993 and 1994 (Boesch and Boesch-Achermann 2000; Muroyama and Sugiyama 1994).

\section{Data Analysis}

Via Microsoft Access 1997 we entered all behavioral data into Taï-ChimpBehaveBase, our large chimpanzee behavior database, and analyzed them on a yearly basis. We included only data from adolescent and adult individuals $\geq 10 \mathrm{yr}$.

Party composition We analyzed data on party composition for $10 \mathrm{yr}$ of observation for females (1992-2001) and 8 yr of observation for males (1992 and 1995-2001). A party comprises all individuals within visibility of the target chimpanzee. The observer moves around the party to ensure this. We noted each change of party composition and calculated the duration of each party. We excluded parties for which we could not identify all individuals. For each individual (Table I) we calculated the percentage of observation time per year that it spent in 1 of the following party types: single-sex, mixed-sex, or lone individuals, which includes mothers traveling

Table I Overview of number of adult and adolescent males and females present at the end of each year

\begin{tabular}{lllllllllll}
\hline & 1992 & 1993 & 1994 & 1995 & 1996 & 1997 & 1998 & 1999 & 2000 & 2001 \\
\hline Male & $6+3$ & $4+3$ & $2+1$ & $2+1$ & $2+1$ & $1+1$ & $2+1$ & $1+1$ & $1+1$ & $1+1$ \\
Female & $15+2$ & $16+3$ & $11+2$ & $11+2$ & $10+2$ & $11+2$ & $11+1$ & $7+0$ & $7+1$ & $6+2$ \\
Com. size & 42 & 41 & 31 & 33 & 33 & 31 & 31 & 22 & 23 & 21 \\
\hline
\end{tabular}

Com. size $=$ community size . The first number refers to adult individuals while the second number refers to adolescent individuals.

Springer 
only with their offspring irrespective of the age of the offspring. Because the sex ratio, which ultimately influences party composition, changed between years, we averaged individual values per year and sex for further analysis. Using yearly values allowed us to reduce the interdependence of data problem, which presents a difficulty when analyzing data of short time periods (Pepper et al. 1999).

Grooming interactions We analyzed data on grooming interactions for $9 \mathrm{yr}$ of observation for females (1993-2001) and 7 yr of observation for males (1995-2001). We recorded only grooming interactions lasting $>1 \mathrm{~min}$. We treated mutual grooming as 2 grooming events, 1 for A grooms B and 1 for B grooms A. We excluded motheroffspring grooming. We defined 3 grooming categories: male-male, female-female, and mixed-sex. We further subdivided mixed-sex grooming into female-male grooming if the actor was female and male-female grooming if the actor was male. For each category we calculated grooming frequencies and average grooming bout length per individual and year. A grooming bout ended when the actor stopped grooming for $>30 \mathrm{~s}$. We calculated frequencies for each chimpanzee as the number of grooming events per $\mathrm{h}$ of focal observation time. Because grooming frequencies are highly dependent on the number of available partners, we compared the observed frequencies with expected frequencies per category based on the average community composition per year, assuming an even distribution of grooming events across all dyads. We calculated grooming bout length as the average grooming duration per category and individual in a given year.

Community size and composition can affect grooming behavior, so that sex differences might be due to differences in demographic variables. Most chimpanzee communities have a highly female-biased sex ratio and females might simply not have enough time to groom all available same-sex partners. For males, which usually have fewer same-sex partners available, time constraints may be less strong. Thus, females, could, e.g., reduce the time they invest in each grooming session to increase their number of grooming partners, which in turn might result in shorter grooming bout length. Alternatively, females could concentrate on fewer partners. We wanted to know if male and female chimpanzees generally differ in the number of same-sex partners they groom or if the reported differences in grooming behavior can be a consequence of differing numbers of same-sex partners. Therefore, we counted the number of same-sex partners that were groomed during each year of observation relative to the number of partners available. Because observation time for females differed between individuals and because an increase in observation time may result in an increase of grooming partners, the data for females represent minimum numbers of grooming partner. One can consider estimates for males as accurate because observation time was usually higher and males usually groomed all other same-sex partners within the community, thereby reaching the maximum number of partners. Because the number of adult males in Taï is too low to allow us to compare the number of grooming partners for males and females under similar conditions, we also included all published data on chimpanzee same-sex grooming partner numbers. This increased the variability of available partners, especially in males, and allowed us to compare number of grooming partners for males and females when similar numbers of partners were available for both sexes. 
Social dyadic association index (sDAI) We calculated dyadic association indices from party membership data for $10 \mathrm{yr}$ of observation (1992-2001). We excluded the generally high sDAIs between mothers and offspring. The index measures the proportion of time that individuals A and B spend together in the same party as compared to the time they spend apart but together with other individuals (Cairns and Schwager 1987). We calculated it as follows:

$$
\mathrm{sDAI}=\frac{\text { social time }_{\mathrm{A}+\mathrm{B} \text { together }}}{\text { social time }_{\mathrm{A} \text { without } \mathrm{B}}+\text { social time }_{\mathrm{B} \text { without } \mathrm{A}}+\text { social time }_{\mathrm{A}+\mathrm{B} \text { together }}}
$$

Contrary to previous uses of the DAI, we included only the social time, i.e., the time not spent alone, of each individual to account for individual differences in their tendency to socialize (Pepper et al. 1999). We calculated values for each dyad and averaged them for male-male, female-female, and mixed-sex dyads on a yearly basis.

Because chimpanzee communities are usually female-biased, average DAI values might be masking existing strong female-female relationships: assuming that chimpanzees cannot maintain close associations with all community members, there will be a higher number of loose associations in the more abundant sex, which in turn reduces the average value, even if a small proportion of the associations are very strong. In the less abundant sex, the same number of high DAI values will have a stronger influence on the average as there are proportionally fewer loose associations. Thus, even if both sexes have some close associates, the higher proportion of loosely associated dyads will reduce average sDAI values in the more abundant sex, i.e., in females, which may lead to the described sexual difference in average sDAI. To investigate if similar strong same-sex associations exist in both sexes, we compared maximum sDAI values per year for each of the 3 classes. The comparison indicates if female associations generally can reach the same strength as male associations and therefore demonstrates more accurately the potential of male and female chimpanzees to form strong social bonds without being confounded by the aforementioned disadvantages when using averages.

\section{Statistical Analyses}

Because we were interested only in overall sexual differences over the entire $10 \mathrm{yr}$, we treated each year as an independent datum and compared means across years per class. This is justified by the fact that community size and composition - and thereby the social environment for each individual - changed dramatically across years and the majority of the individuals present in 1992 did not contribute to the data in 2001. Because changes of absolute values per year might be a consequence of changes in community size (e.g., a general increase of sDAI owing to a decrease in community size) and composition, we used primarily more conservative nonparametric statistics, using rank values rather than absolute values, thereby reducing the effects due to general changes in absolute levels. Because sample size is relatively small, all $p$-values are exact. All tests are 2-tailed and significance level is $p<0.05$. We compared groups via Kruskal-Wallis test, Mann-Whitney $U$-test (MWUT), or Wilcoxon signed rank test 
(WSRT). We used Pearson correlation to analyze the effects of demographic changes on party composition.

To examine whether the sexes differ in number of grooming partners depending on how many partners are available, we tested if the slopes of the regression lines differed significantly (Zar 1999). Because the relationship between partners groomed and partners available is not linear but is expected to reach an asymptote with increasing number of available partners, we log-transformed the data, which allowed us to use a linear regression model for the statistics. However, because we derived data not only from our own study but also from the literature, one needs to evaluate the comparison with care because observational protocols vary among study sites.

\section{Results}

\section{Party Composition}

Males spent most of their time in mixed parties (mean \pm SD: $71 \% \pm 11.5 \%, n=8$ ), while females spent equal time in mixed parties (mean \pm SD: $41 \% \pm 8.6 \%, n=8$ ) and allfemale parties (mean \pm SD: $40 \% \pm 11.3 \%, n=8$; Fig. 1 ). Members of both sexes were rarely alone, as evident from the significantly lower percentage of time spent alone versus time spent in any other party type within each sex (MWUT, males, $n_{1 / 2}=8$ : $U_{\text {mixed }}=0 / U_{\text {uni }}=20, p_{\text {mixed }}=0.001 / p_{\text {uni }}=0.208$, females, $n_{1 / 2}=10: \quad U_{\text {mixed }}=0 / U_{\text {uni }}=2$, $\left.p_{\text {mixed\&uni }}=0.000\right)$. However, in terms of sexual differences, statistical analysis reveals that males spent significantly more time than females in mixed sex parties (MWUT, $U=1, p=0.001, n_{1 / 2}=10 / 8$ ) and significantly less time in unisex parties (MWUT, $U=4$, $p=0.001, n_{1 / 2}=10 / 8$ ) or alone (MWUT, $U=17, p=0.41, n_{1 / 2}=10 / 8$ ). While the amount of time spent in mixed-sex parties is, in the case of the males, dependent on community composition, i.e., the number of partners available within each category (Table II), time spent alone does not correlate significantly with any of the demographic variables for either males or females (Table II). Because we encountered individuals regularly (even more so when community size was low) and did not normally locate targets via acoustics, we believe that there is no strong bias toward observing more social individuals. The fact that time alone remained fairly constant throughout time, despite decreasing community size, supports this inference.

Fig. 1 Mean percentage of total observation time male and female chimpanzees spent either alone (or with their dependent offspring in the case of females), in single-sex parties or in mixed sex parties. Values are averages across 8 and $10 \mathrm{yr}$ of observation, respectively.

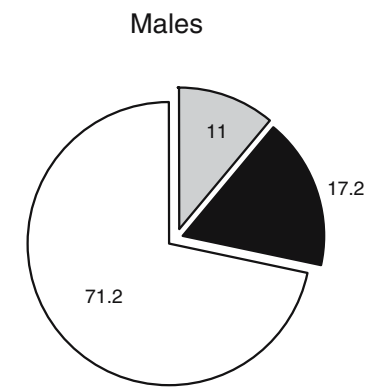

\section{Females}

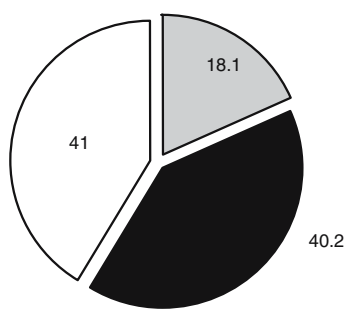


Table II Correlations of demographic variables and party composition for male and female chimpanzees

\begin{tabular}{|c|c|c|c|c|c|c|c|}
\hline & & \multicolumn{3}{|l|}{ Females } & \multicolumn{3}{|l|}{ Males } \\
\hline & & Alone & Unisex & Mixed & Alone & Unisex & Mixed \\
\hline \multirow[t]{3}{*}{ Community size } & $r$ & -0.239 & -0.349 & -0.354 & 0.248 & 0.488 & $-0.791^{*}$ \\
\hline & $p$ & 0.506 & 0.322 & 0.316 & 0.553 & 0.219 & 0.019 \\
\hline & $N$ & 10 & 10 & 10 & 8 & 8 & 8 \\
\hline \multirow[t]{3}{*}{ Adult females } & $r$ & -0.390 & 0.424 & -0.296 & 0.366 & 0.213 & -0.585 \\
\hline & $p$ & 0.265 & 0.223 & 0.406 & 0.373 & 0.613 & 0.128 \\
\hline & $N$ & 10 & 10 & 10 & 8 & 8 & 8 \\
\hline \multirow[t]{3}{*}{ Adult males } & $r$ & 0.111 & 0.003 & -0.134 & 0.084 & 0.622 & $-.828 *$ \\
\hline & $p$ & 0.761 & 0.994 & 0.712 & 0.844 & 0.100 & 0.011 \\
\hline & $N$ & 10 & 10 & 10 & 8 & 8 & 8 \\
\hline \multirow[t]{3}{*}{ All adults } & $r$ & -0.179 & 0.230 & -0.253 & 0.199 & 0.568 & $-0.842 *$ \\
\hline & $p$ & 0.621 & 0.523 & 0.480 & 0.636 & 0.142 & 0.009 \\
\hline & $N$ & 10 & 10 & 10 & 8 & 8 & 8 \\
\hline
\end{tabular}

$r=$ Pearson's correlation coefficients; $p=p$-values; and $N=$ number of years for various party types and demographic variables. Asterisks indicate significant results.

\section{Grooming Interactions}

Based on overall grooming frequencies, females groomed significantly less than expected (WSRT $z=-2.31, p=0.02, n=9$ ), mixed-sex grooming frequencies did not differ from expected values (WSRT, $z=-0.652, p=0.57, n=9$ ) and males groomed significantly more often than expected (WSRT $z=-2.37, p=0.016, n=7$; Fig. 2). Further, males had significantly higher overall grooming frequencies than females (WSRT, $z=-2.2, p=0.031, n=7$ ) and than mixed-sex dyads (WSRT, $z=-2.03, p=$ $0.047, n=7$ ), while female grooming rates did not differ significantly from those of mixed-sex dyads (WSRT, $z=-1.007, p=0.359, n=9$ ). There is also a sexual difference in the amount of time invested in grooming: on average, females groomed actively for

Fig. 2 Observed and expected grooming frequencies for female-female, mixed sex, and male-male grooming dyads. Values are averages across individuals and years \pm standard deviations; asterisks indicate significant differences.

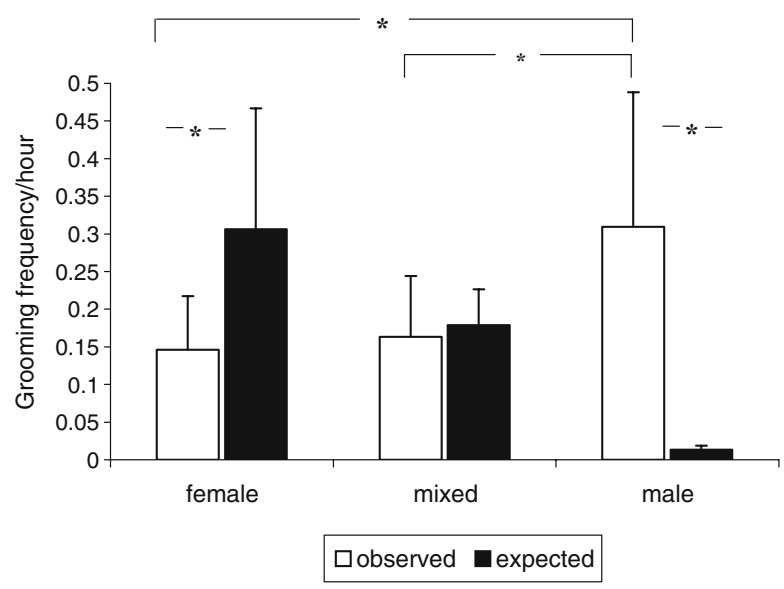


shorter periods than males did, i.e., male grooming bouts lasted longer than female grooming bouts (Kruskal-Wallis: $\chi^{2}=9.93 \mathrm{df}=3 p<0.02$; posthoc MWUT: $\mathrm{f}-\mathrm{f} / \mathrm{m}-\mathrm{m} U=$ $12, n_{1 / 2}=9 / 7, p=0.042 ; \mathrm{f}-\mathrm{m} / \mathrm{m}-\mathrm{m} U=10, n_{1 / 2}=9 / 7, p=0.023$; f-f $/ \mathrm{m}-\mathrm{f} U=18, n_{1 / 2}=9 / 9$, $p=0.05$; $\left.\mathrm{f}-\mathrm{m} / \mathrm{m}-\mathrm{f} U=14, n_{1 / 2}=9 / 9, p=0.019\right)$, indicating that they generally spent more time grooming their partners than females did (Fig. 3).

Figure 4 contains the mean number of same-sex grooming partners in relation to the number of available same-sex partners using data from Taï and from published findings from other study sites. For Taï, we depict 1 value per year, because the number of available partners changed across years. Males and females groom a similar number of partners when we control for the number of partners available (comparison of regression slopes: $n=32, \mathrm{df}=28, t=-0.212$, n.s.). A possible underestimation for females grooming partners due to shorter observation times versus that for males therefore favors our argument that males and females do not differ in the number of partners groomed when controlling for the number of available partners.

\section{Social Dyadic Association Index}

Average sDAI differs significantly between the sexes (Kruskal-Wallis: $\chi^{2}=7.53, \mathrm{df}=$ $2, p=0.025)$. It is significantly higher in male dyads compared to mixed-sex (MWUT, $U=18, p=0.016, n_{1 / 2}=10 / 10$ ) and female dyads (MWUT, $U=21, p=0.028$, $\left.n_{1 / 2}=10 / 10\right)$. There is no significant difference between females and mixed-sex dyads (MWUT, $U=44, p=0.65, n_{1 / 2}=10 / 10$ ), indicating that females associate with each other to the same extent as with males (Fig. 5a). However, maximum sDAIs reveal that both males and females can have favored association partners with which they reach very high sDAIs. Though the overall sex difference is significant (Kruskal-Wallis: $\chi^{2}=11.08, \mathrm{df}=2, p=0.004$ ), this is due to the significantly lower sDAI of mixed-sex dyads versus unisex dyads (males: MWUT, $U=21, p=0.028$, $n_{1 / 2}=10 / 10$; females: MWUT, $U=4, p=0.001, n_{1 / 2}=10 / 10$ ), indicating that no association between the sexes reached the same extent as same-sex associations did. There is no significant difference in maximum sDAIs between males and females (MWUT, $U=50, p=1, n_{1 / 2}=10 / 10$ ). A typical social network of the Taï chimpanzees based on their sDAIs in 1993 is in Fig. 6a and b. The strongest associations (sDAI 0.5-1; thickest lines in Fig. 6a) are those between mothers and their adolescent sons

Fig. 3 Average grooming bout duration for different actorreceiver dyads. Error bars are standard errors across the $10 \mathrm{yr}$ of observation. All sexual differences are significant.

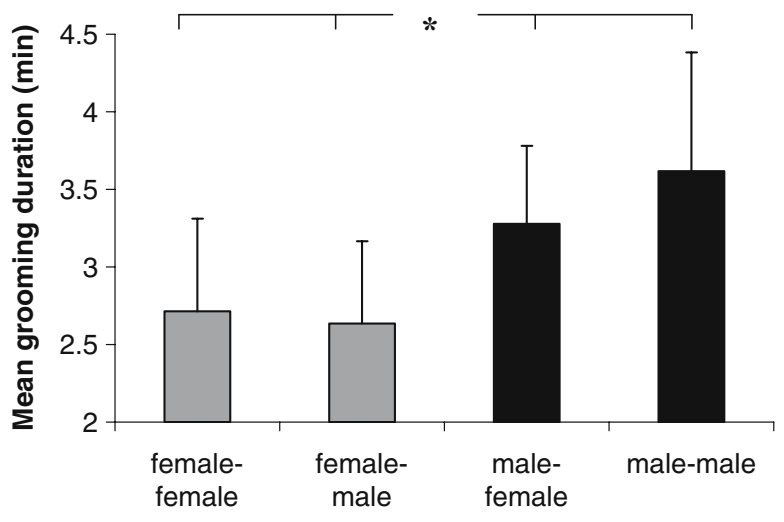




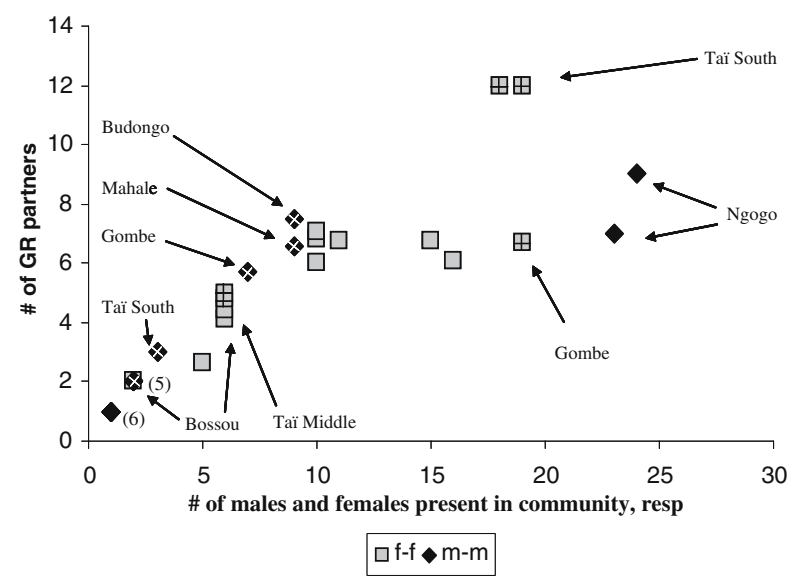

Fig. 4 Average number of same-sex grooming partners for male and female chimpanzees within and across study sites, depending on the number of partners available. There is no significant sexual difference. Data from the Taï North community are depicted as simple filled symbols (like those in the legend), while data from other study sites are marked with an additional white cross (for females) or with a black plus (for males). We derived the data from Muroyama and Sugiyama (1994) for Gombe, Bossou, and Mahale; from Watts (2000a) for Ngogo; from Arnold and Whiten (2003) for Budongo; and from Lehmann and Boesch (unpublished data) for Taï South and Middle. Numbers in parentheses refer to the number of data points at the respective place.

(Fan-Mar and Git-Gip) and those between certain unrelated female dyads/triads (BijMys-Pou, Ven-Her, Lou-Cho, whereas Cho is an adolescent female whose mother was Lou's closest association partner before her death). Only at the next level do associations between pairs of males occur (sDAI 0.30-0.49; dashed lines in Fig. 6a), followed by further same-sex and mixed-sex associations on the lowest level $(0.15$ and 0.29 ; thin lines, Fig. $6 \mathrm{~b}$ ). Thus, interfemale relationships are among the strongest relations within the community.

\section{Discussion}

Compared to our knowledge of male chimpanzee sociality little is known about female social relationships. Our data support the previous findings that males and females clearly differ in some measures of sociality (Goodall 1986; Nishida 1990; Wrangham 1986): males are alone less often, groom each other more often and for longer spans, and generally have a higher average sDAI. The observations are compatible with predictions derived from dispersal patterns and kin selection theory and some models of primate social evolution (Isbell and Young 2002; Sterck et al. 1997; van Schaik 1989; Wrangham 1980). However, despite the clear and significant sexual differences, our data also demonstrate that Taï chimpanzee females cannot be regarded as an asocial sex. They show a high degree of sociality: spending $82 \%$ of their time in parties with other individuals, often other adult females (Fig. 1), establishing grooming relationships with a number of same-sex partners comparable to males, and having similarly high sDAIs, indicating that some females form strong associations with other females (Boesch and Boesch-Achermann 2000). 
Fig. 5 (a) Average social dyadic association index for female, male, and mixed-sex dyads with standard deviation across $10 \mathrm{yr}$ of observation. Asterisks indicate significant differences. (b) Maximum value of the social dyadic association index for female, male, and mixed-sex dyads with standard deviation across $10 \mathrm{yr}$ of observation. Asterisks indicate significant differences.
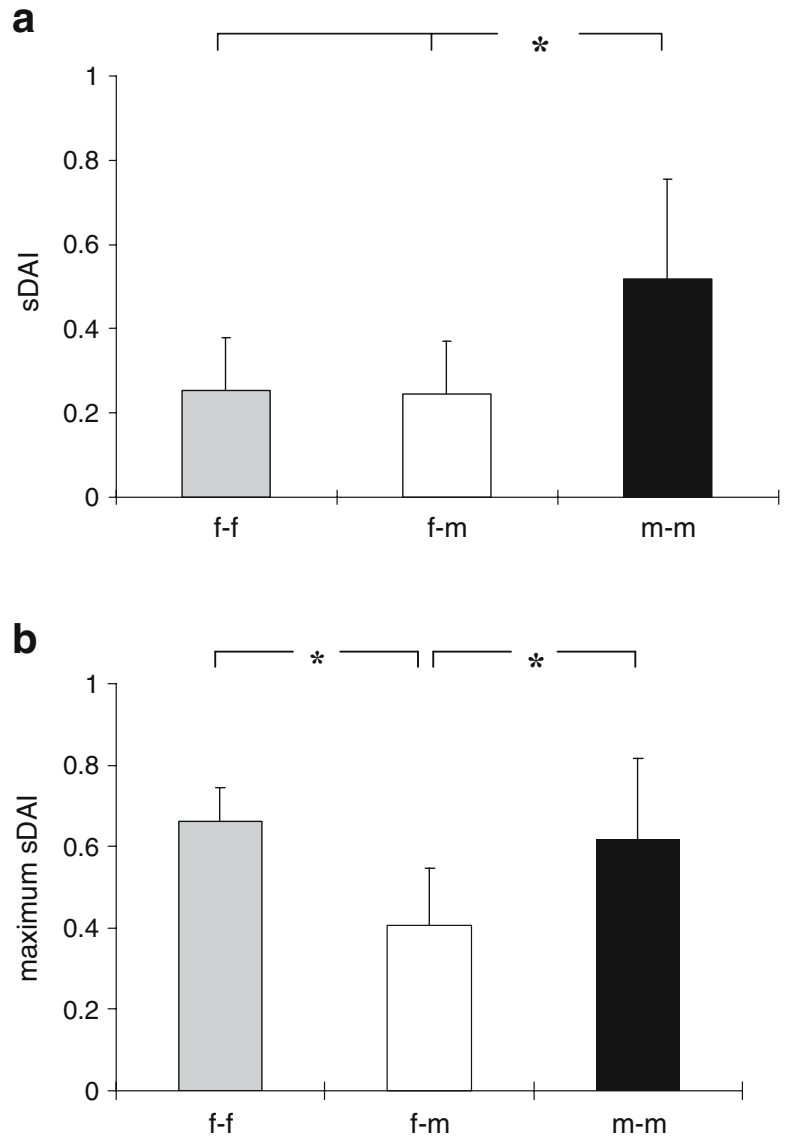

If females in Taï are highly social, why have other researchers concluded that chimpanzee females are generally asocial? First, most chimpanzee studies were concentrated on male behavior (Arnold and Whiten 2003; Furuichi and Ihobe 1994; Mitani et al. 2000, 2002; Nishida 1990;Watts 2000a,b, 2002; Watts et al. 2000), and observations on females were often a side product when they were observed in association with males (Arnold and Whiten 2003), which may generally lead to an underestimation of female sociality. Further, females are often difficult to habituate, so that their sociality may be observed only much later, after all females are well habituated to the presence of humans. However, as more researchers concentrate on chimpanzee female social relationships (Fawcett 2000; Williams et al. 2002b), evidence increases that they can display relatively high degrees of social behavior (Fawcett 2000; Matsumoto-Oda 2002; Sugiyama 1988; Sugiyama and Koman 1979). Third, the notion of asocial females is usually based on the parameters for which we also found significant sexual differences. However, because many chimpanzee communities have a largely biased sex ratio it may be more revealing to calculate the actual degree of sexual differences, rather than to analyze whether average values differ, and to increase the number of parameters to measure sociality. 
Fig. 6 Sociogram of all Taï chimpanzees $>10$ yr of age in 1993 , based on social dyadic association indices (sDAI). Males are represented as black triangles, females as gray circles. Thickness of lines represents the strength of dyadic association with thick lines (a) depicting all associations between 0.50 and 1 , dashed lines (a) depicting sDAIs between 0.30 and 0.49 , and thin lines (b) depicting sDAIs between 0.15 and 0.29. Classification follows Goodall (1986). Relationships between mothers and their adolescent sons and females and an adopted daughter are marked.
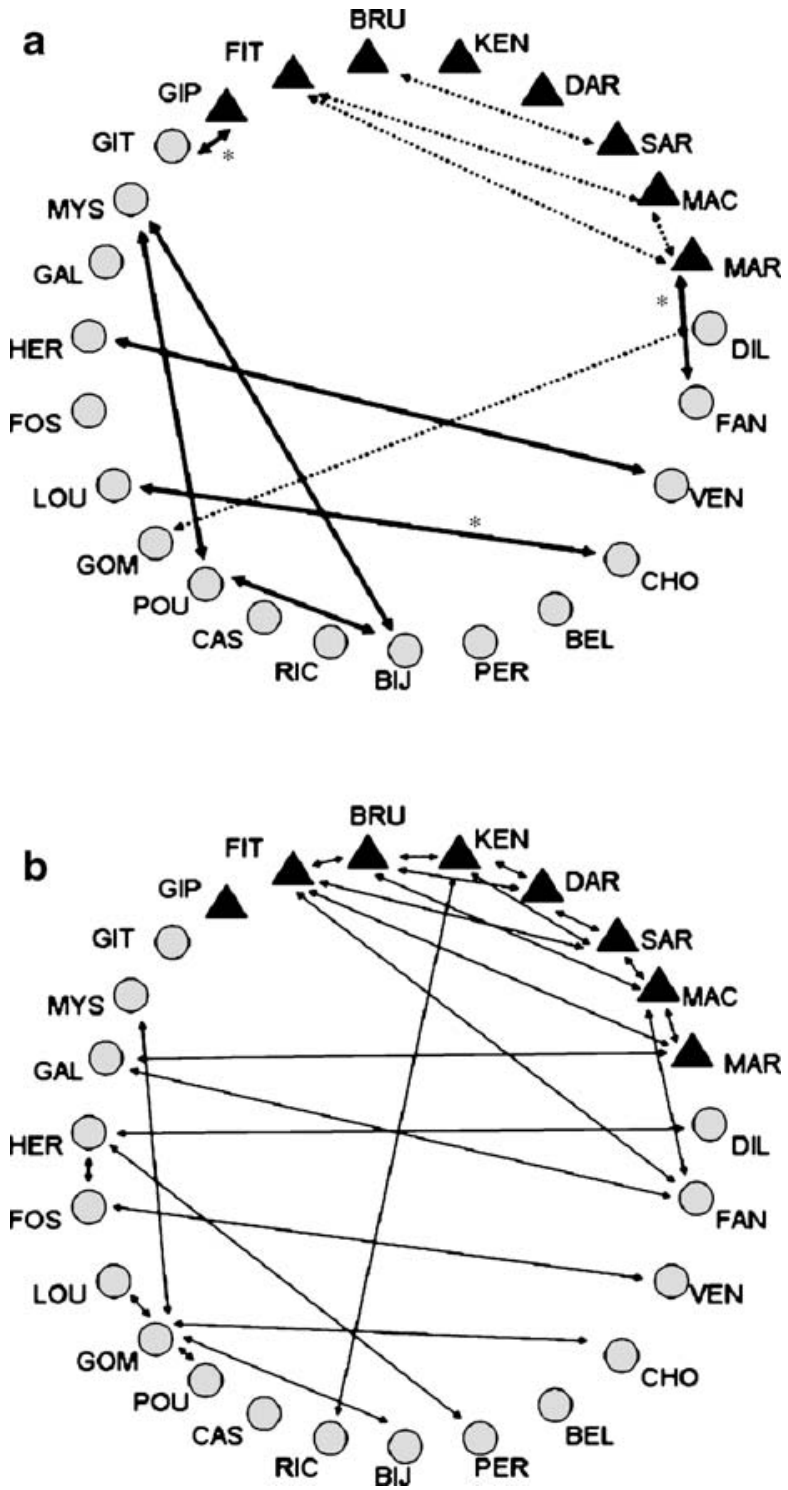

In communities with highly biased sex ratios, it may not be a good approach to compare only average values of affiliative behaviors because of the stronger dilution effect in the more abundant sex. Thus, simply analyzing averages may distort the overall picture by underestimating the degree of sociality in the more frequent sex.

A second reason why researchers have not found high sociality for all female chimpanzees might be that grouping patterns themselves are highly dependent on group size and composition (Lehmann and Boesch 2004) as well as on ecological parameters (Anderson et al. 2002) and the resulting levels of intrasexual competition. Socio-ecological models predict that sociality is high when intrasexual competition is high (Sterck et al. 1997), though some others have suggested that the 
reverse should be true, i.e., strong competition within 1 sex leads to weak social relationships between same-sex individuals (Hemelrijk and Luteijn 1998). Intense feeding competition among chimpanzees may lead to high costs of social behavior, so that chimpanzees, which suffer less predation risk than smaller monkeys do, will reduce competition by staying more solitary or only with their dependent offspring. However, some researchers have hypothesized that high contest competition actually favors strong social bonds, so that individuals form coalitions and cooperate to defend resources. Chimpanzee males may face strong competition over reproduction, leading to the generally observed high level of sociality among males, while females experience mainly feeding competition, the extent of which may differ between sites. Females in Taï may face stronger competition than females at other study sites do, leading to the observed pattern of sociality. Wittig and Boesch (2003) have shown that, contrary to other chimpanzee populations, Taï chimpanzee females form linear hierarchies, which usually is regarded to be a mechanism to reduce high levels of competition. Thus, though the Taï National Park in general may be a relatively rich environment with low food competition, especially when compared to Gombe, Taï chimpanzee females may still experience high intrasexual levels of competition (Wittig and Boesch 2003). But it remains open whether this is a consequence of high levels of sociality due to, e.g., high predation pressure or clumped food resources or the cause of the nature of female relationships, assuming that sociality has other major fitness consequences apart from reducing food competition. Recently, Silk et al. (2003) showed that socially well-integrated female baboons have higher infant survival, demonstrating for the first time the adaptive value of sociality, independent of other parameters such as dominance rank and environmental factors. One can hypothesize that sociality has a similar beneficial effect in other species, including chimpanzees. In favor of this are observations of cases wherein a mother that had a young offspring died while her offspring survived: her offspring was "adopted" by the mothers' closest or second to closest association partner ( $n=4$ of 5 cases). More direct additional benefits of long-term associations between individual females may lie in socialization of offspring (Williams et al. $2002 \mathrm{~b}$ ) or in support during aggressive interactions, which might be particularly important for females if they have a linear dominance hierarchy (Boesch and Boesch-Achermann 2000; Wittig and Boesch 2003) and if reproductive success is linked to dominance (Pusey et al. 1997).

A similar variation in female sociality has been reported by Starin (1994) for red colobus, and by Lusseau et al. (2003) for bottlenose dolphins, though here kin relationships are not yet clear, so that high levels of association could potentially be due to alliances between kin, which is not the case in Taï chimpanzees. Researchers have usually attributed sexual differences in chimpanzee sociality to the presumed high degree of relatedness between males as compared to females; however, the hypothesis has been weakened since Vigilant et al. (2001) showed that males on average are not more closely related than females. Further, data from Kanyawara, Ngogo, and Taï indicate that it is not primarily related dyads that cooperate with each other, and levels of relatedness do not predict levels of cooperation between males in wild chimpanzee communities (Boesch et al. 2006; Goldberg and Wrangham 1997; Mitani et al. 2000). Similarly, in female bonobos relatedness is a poor predictor of the formation of social bonds (Hashimoto et al. 1996). Thus, sociality is not strictly 
governed by relatedness, and one cannot always explain the existence of social bonds via kin-selection and dispersal patterns. Further, chimpanzees are long-lived and both males and females spent their entire adult lives in 1 community (secondary dispersal is rare), with both sexes having (linear) dominance hierarchies, so one should expect them to benefit in a similar way from social interactions and cooperation. Like males, females could use the help of allies to climb in the hierarchy and to gain access to resources, especially when female reproductive success is linked to female dominance (Pusey et al. 1997). However, sexual differences in sociality have been described for all chimpanzee communities studied (Boesch and Boesch-Achermann 2000; Fawcett 2000; Goodall 1986; Matsumoto-Oda 2002; Wrangham and Smuts 1980; Wrangham et al. 1992), indicating that males may gain more from the behaviors or that levels of intrasexual competition are different (presumably also depending on sex ratios), thereby leading to different behavioral outcomes. In addition, male and female social affiliations might have fundamentally different functions. While females form longterm bonds that can last over many years (up to 6 consecutive years: Boesch and Boesch-Achermann 2000), males seem to have more frequently changing association partners (Lehmann and Boesch, unpubl. data), suggesting that close association partners for males may be more influenced by intragroup politics while females form long-term bonds with other females which often (but not necessarily) have same-aged offspring.

Our finding of high female sociality despite significant sexual differences has strong implications for recent models of primate evolution of sociality (Isbell and Young 2002; Sterck et al. 1997; van Schaik 1989; Wrangham 1980), which are all based on the assumption that females experience levels of competition that are high enough to favor the development of strong social bonds only in female-philopatric species. For species with female dispersal, most authors conclude that female social relationships and hierarchies should be generally weak or nonexistent, and chimpanzees have repeatedly been classified as dispersal egalitarian (Isbell and Young 2002; van Schaik 1989; Sterck et al. 1997; Wrangham 1980). However, as more data on female relationships accumulate, it becomes evident that chimpanzee female sociality can vary dramatically between populations, indicating that one cannot easily use the classification systems on a species level. One should consider a reclassification of chimpanzees alongside an extension of the models to accommodate variability in social behaviour accurately ( $c f$. Williams et al. 2002b). Lifehistory parameters, such as a long life within a stable community after dispersal, female dominance hierarchies, and long periods of infant dependency seem to favor female social relationships, as in chimpanzees and bonobos (Kano 1992).

In summary, our study adds to the growing body of evidence that sociality in primates is not always explained by patterns of relatedness and kin selection theory. We have shown that although significant sexual differences in sociality exist, there is high sociality in both sexes, and the notion of the asocial chimpanzee female has to be revised because female associations can be among the strongest in the chimpanzee social network (Fig. 6). Our data suggest that in chimpanzees adult females can be very social but that the degree of sociality varies between study sites. Existing models of primate sociality should therefore incorporate the possibility that the dispersing sex can also have refined social relationships. 
Acknowledgments We thank the Ministère de la Recherche Scientifique, the Ministère de l'Agriculture et des Ressources Animales, and the direction of the Taï National Park of Côte d'Ivoire for permitting the research in the Taï National Park. We thank the Centre Suisse in Abidjan for their support. We thank the Fonds National Suisse de Recherche Scientifique and the Max Planck Society for financial support. We also thank the field assistants Grégoire Kohou Nohon and Honora Néné Kpazahi, who collected the data provided here. Finally, we thank Maik Thraenert and Alexander Burkhard for their help with the database creation; 6 student assistants for typing in all the data; Linda Vigilant, Laurent Lacroix, Brenda Bradley, and Daniel Stahl for valuable discussions and social support.

\section{References}

Altmann, J. (1974). Observational study of behavior: Sampling methods. Behaviour, 49, 227-267.

Anderson, D. P., Nordheim, E. V., Boesch, C., \& Moermond, T. C. (2002). Factors influencing fissionfusion grouping in chimpanzees in the Taï National Park, Côte d'Ivoire. In C. Boesch, G. Hohmann, \& L. Marchant (Eds.) Behavioral diversity in chimpanzees and bonobos (pp. 90-101). Cambridge, U. K.: Cambridge University Press.

Arnold, K., \& Whiten, A. (2003). Grooming interactions among the chimpanzees of the Budongo forest, Uganda: Tests of five explanatory models. Behaviour, 140, 519-552.

Boesch, C., Boesch, H., \& Vigilant, L. (2006). Cooperative hunting in chimpanzees: Kinship or mutualism? In P. M. Kappeler, \& C. P. van Schaik (Eds.) Cooperation in primates and humans: Mechanisms and evolution (pp. 139-150). Berlin: Springer-Verlag.

Boesch, C., \& Boesch-Achermann, H. (2000). The Chimpanzees of the Taï Forest: Behavioural ecology and evolution. Oxford: Oxford University Press.

Cairns, S. J., \& Schwager, S. J. (1987). A comparison of association indices. Animal Behavior, 35, 1454 1469.

Clutton-Brock, T. (2002). Breeding together: Kin selection and mutualism in cooperative vertebrates. Science, 296, 69-72.

Dunbar, R. I. M. (1984). Reproductive decisions: An economic analysis of Gelada baboon social strategies. Princeton, NJ: Princeton University Press.

Fawcett, K. A. (2000). Female relationships and food availability in a forest community of chimpanzees. $\mathrm{PhD}$ thesis, University of Edinburgh.

Furuichi, T., \& Ihobe, H. (1994). Variation in male relationships in bonobos and chimpanzees. Behaviour, 130, 211-228.

Ghiglieri, M. P. (1984). Feeding ecology and sociality of chimpanzees in Kibale Forest, Uganda. In P. S. Rodman, \& J. G. H. Cant (Eds.) Adaptations for foraging in nonhuman primates (pp. 161-194). New York: Columbia University Press.

Goldberg, T. L., \& Wrangham, R. W. (1997). Genetic correlates of social behaviour in wild chimpanzees: Evidence from mitochondrial DNA. Animal Behavior, 54, 559-570.

Goodall, J. (1986). The chimpanzees of Gombe: Patterns of behavior. Cambridge, MA: The Belknap Press of Harvard University Press.

Greenwood, P. J. (1980). Mating systems, philopatry and dispersal in birds and mammals. Animal Behavior, 28, 1140-1162.

Hamilton, W. D. (1964). The genetic evolution of social behaviour I, II. Journal of Theoretical Biology, 7 , $1-52$.

Harcourt, A. H., \& Stewart, K. J. (1983). Interactions, relationships, and social strucutre: The great apes. In R. A. Hinde (Ed.) Primate social relationships: An integrated approach (pp. 307-314). Oxford: Blackwell.

Hashimoto, C., Furuichi, T., \& Takenaka, O. (1996). Matrilineal kin relationship and social behavior of wild bonobos (Pan paniscus). Sequencing the D-loop region of mitochondrial DNA. Primates, 37, 305-318.

Hemelrijk, C. K., \& Luteijn, M. (1998). Philopatry, male presence and grooming reciprocation among female primates: A comparative perspective. Behavioral Ecology and Sociobiology, 42, 207-215.

Hohmann, G., Gerloff, U., Tautz, D., \& Fruth, B. (1999). Social bonds and genetic ties: Kinship, association and affiliation in a community of Bonobos (Pan paniscus). Behaviour, 136, 1219-1235.

Huntingford, F. A., \& Turner, A. K. (1987). Animal conflict. London and New York: Chapman and Hall. Isbell, L. A., \& Young, T. P. (2002). Ecological models of female social relationships in primates: Similarities, disparities, and some directions for future clarity. Behaviour, 139, 177-202. 
Kano, T. (1992). The last ape: Pygmy chimpanzee behavior and ecology. Stanford: Stanford University Press.

Kapsalis, E., \& Berman, C. M. (1996). Models of affiliative relationships among free-ranging rhesus monkeys (Macaca mulatta).1. Criteria for kinship. Behaviour, 133, 1209-1234.

Kawanaka, K. (1984). Association, ranging, and the social unit in chimpanzees of the Mahale Mountains, Tanzania. International Journal of Primatology, 5, 411-434.

Kummer, H. (1968). Social organization of Hamadryas baboons. Chicago: University of Chicago Press.

Lehmann, J., \& Boesch, C. (2004). To fission or to fusion: Effects of community size on wild chimpanzee (Pan troglodytes verus) social organisation. Behavioral Ecology and Sociobiology, 56, 207-216.

Lusseau, D., Schneider, K., Boisseau, O. J., Haase, P., Slooten, E., \& Dawson, S. M. (2003). The bottlenose dolphin community of Doubtful Sound features a large proportion of long-lasting associations. Can geographic isolation explain this unique trait? Behavioral Ecology and Sociobiology, 54(4), 396-405.

Matsumoto-Oda, A. (2002). Social relationships between cycling females and adult males in Mahale chimpanzees. In C. Boesch, G. Hohmann, \& L. Marchant (Eds.) Behavioral diversity in chimpanzees and bonobos (pp. 168-180). Cambridge, U.K.: Cambridge University Press.

Mitani, J. C., Merriwether, D. A., \& Zhang, C. B. (2000). Male affiliation, cooperation and kinship in wild chimpanzees. Animal Behavior, 59, 885-893.

Mitani, J. C., Watts, D. P., Pepper, J. W., \& Merriwether, D. A. (2002). Demographic and social constraints on male chimpanzee behaviour. Animal Behavior, 64, 727-737.

Morin, P. A., Moore, J. J., Chakraborty, R., Jin, L., Goodall, J., \& Woodruff, D. S. (1994). Kin selection, social structure, gene flow, and the evolution of chimpanzees. Science, 265, 1193-1201.

Muroyama, Y., \& Sugiyama, Y. (1994). Grooming relationships in two species of chimpanzees. In R. W. Wrangham, W. C. McGrew, F. B. M. de Waal, \& P. Heltne (Eds.) Chimpanzee cultures. Cambridge MA: Harward University Press.

Nishida, T. (1979). The social structure of chimpanzees of the Mahali Mountains. In D. A. M. E. Hamburg (Ed.) The great apes: Perspecting on human evolution (vol. vol. 5, pp. 73-121). Menlo Park, CA: Benjamin/Cummings.

Nishida, T. (1990). The chimpanzees of the Mahale Mountains. Tokyo: University of Tokyo Press.

Nishida, T., Hiraiwa-Hasegawa, M., Hasegawa, T., \& Takahata, Y. (1985). Group extinction and female transfer in wild chimpanzees in the Mahale National-Park, Tanzania. Zeitschrift für Tierpsychologie, 67, 284-301.

Parish, A. R., \& De Waal, F. B. M. (2000). The other "closest living relative". Annals of the New York Academy of Science, 907, 97-113.

Pepper, J. W., Mitani, J. C., \& Watts, D. P. (1999). General gregariousness and specific social preferences among wild chimpanzees. International Journal of Primatology, 20, 613-632.

Perry, S. (1996). Female-female social relationships in wild white-faced capuchin monkeys, Cebus capucinus. American Journal of Primatology, 40, 167182.

Pope, T. R. (2000). Reproductive success increases with degree of kinship in cooperative coalitions of female red howler monkeys (Alouatta seniculus). Behavioral Ecology and Sociobiology, 48, 253-267.

Pusey, A. E., Williams, J. M., \& Goodall, J. (1997). The influence of dominance rank on the reproductive success in female chimpanzees. Science, 277, 828-831.

Seyfarth, R. M. (1976). Social relationships among adult female baboons. Animal Behavior, 24, 917-938.

Seyfarth, R. M. (1980). The distribution of grooming and related behaviors among adult female vervet monkeys. Animal Behavior, 28, 798-813.

Silk, J. B., Alberts, S. C., \& Altmann, J. (2003). Social bonds of female baboons enhance infant survival. Science, 302, 1231-1234.

Silk, J. B., Seyfarth, R. M., \& Cheney, D. L. (1999). The structure of social relationships among female savanna baboons in Moremi Reserve, Botswana. Behaviour, 136, 679-703.

Smuts, B. B. (1990). Sisterhood is powerful: Aggression, competition and cooperation in nonhuman primate societies. In M. Haug, D. Benton, P. F. Brain, B. Olivier, \& J. Mos (Eds.) The aggressive female (pp. 115-136). Den Haag: CIP-Gigevans Koninklijke Bibliotheek.

Starin, E. D. (1994). Philopatry and affiliation among red colobus. Behaviour, 130, 253-270.

Sterck, E. H. M., Watts, D. P., \& van Schaik, C. P. (1997). The evolution of female social relationships in nonhuman primates. Behavioral Ecology and Sociobiology, 41, 291-309.

Struhsaker, T. T., \& Leland, L. (1976). Socioecology of five sympatric monkey species in the Kibale Forest, Uganda. New York: Academic Press.

Sugiyama, Y. (1988). Grooming interactions among adult chimpanzees in Bossou, Guinea, with special reference to social structure. International Journal of Primatology, 9, 393-407. 
Sugiyama, Y., \& Koman, J. (1979). Social structure and dynamics of wild chimpanzees in Bossou, Guinea. Primates, 20, 323-339.

Swedell, L. (2002). Affiliation among females in wild Hamadryas baboons (Papio hamadryas hamadryas). International Journal of Primatology, 23, 1205-1226.

van Schaik, C. P. (1989). The ecology of social relationships amongst female primates. In V. Standen, \& R. A. Foley (Eds.) Comparative socioecology: The behavioural ecology of humans and other mammals (pp. 195-218). Oxford: Blackwell.

Vigilant, L., Hofreiter, M., Siedel, H., \& Boesch, C. (2001). Paternity and relatedness in wild chimpanzee communities. Proceedings of the National Academy of Sciences of the United States of America, 98, $12890-12895$.

Wakefield, M. (2002). Female chimpanzee allogrooming behavior at Ngogo, Kibale National Park, Uganda. American Journal of Physical Anthropology (Supplement), 34, 160-161.

Waser, P. (1988). Resources, philopatry, and social interactions among mammals. In C. N. Slobodchikoff (Ed.) The ecology of social behavior (pp. 109-130). San Diego: Academic Press.

Watts, D. P. (2000a). Grooming between male chimpanzees at Ngogo, Kibale National Park. I. Partner number and diversity and grooming reciprocity. International Journal of Primatology, 21, 189-210.

Watts, D. P. (2000b). Grooming between male chimpanzees at Ngogo, Kibale National Park. II. Influence of male rank and possible competition for partners. International Journal of Primatology, 21, 211238.

Watts, D. P. (2002). Reciprocity and interchange in the social relationships of wild male chimpanzees. Behaviour, 139, 343-370.

Watts, D. P., Colmenares, F., \& Arnold, K. (2000). Redirection, consolation, and male policing. In F. Aureli, \& F. B. M. de Waal (Eds.) Natural conflict resolution (pp. 281-301). London: University Press.

Watts, D. P., \& Mitani, J. C. (2001). Boundary patrols and intergroup encounters in wild chimpanzees. Behaviour, 138, 299-327.

Williams, J. M., Hsien-Yang, L., \& Pusey, A. E. (2002b). Costs and benefits of grouping for female chimpanzees at Gombe. In C. Boesch, G. Hohmann, \& L. F. Marchant (Eds.) Behavioural diversity in chimpanzees and bonobos (pp. 192-203). Cambridge: Cambridge University Press.

Williams, J. M., Oehlert, G. W., Carlis, J. V., \& Pusey, A. E. (2004). Why do male chimpanzees defend a group range? Animal Behaviour, 68, 523-532.

Williams, J. M., Pusey, A. E., Carlis, J. V., Farm, B. P., \& Goodall, J. (2002a). Female competition and male territorial behaviour influence female chimpanzees' ranging patterns. Animal Behavior, 63, 347360 .

Wittig, R. M., \& Boesch, C. (2003). Food competition and linear dominance hierarchy among female chimpanzees in the Taï National Park. International Journal of Primatology, 24, 847-867.

Wrangham, R. W. (1980). An ecological model of female-bonded primate groups. Behaviour, 75, 262300 .

Wrangham, R. W. (1986). Social relationships in chimpanzees. In D. I. Rubenstein, \& R. W. Wrangham (Eds.) Ecological aspects of social evolution: Birds and mammals (pp. 352-378). New Jersey: Princton University Press.

Wrangham, R. W., Clark, A. P., \& Isabirye-Basuta, G. (1992). Female social relationships and social organisation of the Kibale Forest chimpanzees. In T. Nishida, W. C. McGrew, P. Marler, M. Pickford, \& F. D. M. de Waal (Eds.) Topics in primatology, Human origins (vol. 1, pp. 81-98). Tokyo: University of Tokyo Press.

Wrangham, R. W., \& Smuts, B. B. (1980). Sex differences in the behavioural ecology of chimpanzees in the Gombe National Park, Tanzania. Journal of Reproduction and Fertility (Supplement), 28, 13-31.

Zar, J. H. (1999). Biostatistical analysis. Upper Saddle River, NJ: Prentice Hall. 